\title{
DIRECT AND INVERSE THEOREMS OF APPROXIMATION THEORY FOR A GENERALISED MODULUS OF SMOOTHNESS
}

\author{
M. K. POTAPOV AND F. M. BERISHA
}

\begin{abstract}
An asymmetric operator of generalised translation is introduced in this paper. Using this operator, we define a generalised modulus of smoothness and prove direct and inverse theorems of approximation theory for it.
\end{abstract}

\section{Introduction}

In a number of papers (see, e.g., [1, 3, 6, 8, ) direct and inverse theorems of approximation theory are proved for generalised moduli of smoothness defined by means of symmetric operators of generalised translation. It is of interest to obtain the same results for a moduli of smoothness defined by means of asymmetric operators of generalised translation.

In the present paper such an operator is introduced, the generalised modulus of smoothness is defined by its means, and direct and inverse theorems of approximation theory are proved for that modulus.

1. By $L_{p}$ we denote the set of functions $f$ such that in the case $1 \leq p<\infty, f$ is measurable on the segment $[-1,1]$ and

$$
\|f\|_{p}=\left(\int_{-1}^{1}|f(x)|^{p} d x\right)^{1 / p}<\infty
$$

and in the case $p=\infty$, the function $f$ is continuous on the segment $[-1,1]$, and

$$
\|f\|_{\infty}=\max _{-1 \leq x \leq 1}|f(x)| .
$$

Denote by $L_{p, \alpha}$ the set of functions $f$ such that $f(x)\left(1-x^{2}\right)^{\alpha} \in L_{p}$, and put

$$
\|f\|_{p, \alpha}=\left\|f(x)\left(1-x^{2}\right)^{\alpha}\right\|_{p} .
$$

By $E_{n}(f)_{p, \alpha}$ we denote the best approximation of the function $f \in L_{p, \alpha}$ by algebraic polynomials of degree not greater than $n-1$, in $L_{p, \alpha}$ metrics, i.e.,

$$
E_{n}(f)_{p, \alpha}=\inf _{P_{n} \in \mathbb{P}_{n}}\left\|f-P_{n}\right\|_{p, \alpha},
$$

where $\mathbb{P}_{n}$ is the set of algebraic polynomials of degree not greater than $n-1$.

By $D_{x, \nu, \mu}$ we denote the operator

$$
D_{x, \nu, \mu}=\left(1-x^{2}\right) \frac{d^{2}}{d x^{2}}+(\mu-\nu-(\nu+\mu+2) x) \frac{d}{d x} .
$$

It is obvoious that

$$
D_{x, \nu, \mu}=(1-x)^{-\nu}(1+x)^{-\mu} \frac{d}{d x}(1-x)^{\nu+1}(1+x)^{\mu+1} \frac{d}{d x} .
$$

1991 Mathematics Subject Classification. Primary 41A35, Secondary 41A50, 42A16.

Key words and phrases. Generalised modulus of smoothness, asymmetric operator of generalised translation, Jackson theorem, converse theorem, best approximations by algebraic polynomials.

This work was done under the support of the Russian Foundation for Fundamental Scientific Research, Grant \#97-01-00010 and Grant \#96/97-15-96073. 
We say that $g(x) \in A D(p, \alpha)$ if $g(x) \in L_{p, \alpha}$, the derivative $g^{\prime}(x)$ is absolutely continuous on every segment $[a, b] \subset(-1,1)$, and $D_{x, 2,2} g(x) \in L_{p, \alpha}$.

Let

$$
K(f, \delta)_{p, \alpha}=\inf _{g \in A D(p, \alpha)}\left(\|f-g\|_{p, \alpha}+\delta^{2}\left\|D_{x, 2,2} g(x)\right\|_{p, \alpha}\right)
$$

denote the $K$-functional of Peetre interpolating between spaces $L_{p, \alpha}$ and $A D(p, \alpha)$.

We define the operator of generalised translation $\hat{\tau}_{t}(f, x)$ by

$$
\begin{aligned}
\hat{\tau}_{t}(f, x) & =\frac{1}{\pi\left(1-x^{2}\right) \cos ^{4} t / 2} \\
\times & \int_{0}^{\pi}\left(2\left(\sqrt{1-x^{2}} \cos t+x \sin t \cos \varphi+\sqrt{1-x^{2}}(1-\cos t) \sin ^{2} \varphi\right)^{2}\right. \\
- & \left.+\left(x \cos t-\sqrt{1-x^{2}} \sin t \cos \varphi\right)^{2}\right) f\left(x \cos t-\sqrt{1-x^{2}} \sin t \cos \varphi\right) d \varphi .
\end{aligned}
$$

By means of the operator of generalised translation, for a function $f \in L_{p, \alpha}$, we define the generalised modulus of smoothness as follows

$$
\hat{\omega}(f, \delta)_{p, \alpha}=\sup _{|t| \leq \delta}\left\|\hat{\tau}_{t}(f, x)-f(x)\right\|_{p, \alpha} .
$$

Put $y=\cos t, z=\cos \varphi$ in the operator $\tau_{t}(f, x)$, we denote it by $\tau_{y}(f, x)$ and rewrite it in the form

$$
\tau_{y}(f, x)=\frac{4}{\pi\left(1-x^{2}\right)(1+y)^{2}} \int_{-1}^{1} B_{y}(x, z, R) f(R) \frac{d z}{\sqrt{1-z^{2}}}
$$

where

$$
\begin{gathered}
R=x y-z \sqrt{1-x^{2}} \sqrt{1-y^{2}} \\
B_{y}(x, z, R)=2\left(\sqrt{1-x^{2}} y+z x \sqrt{1-y^{2}}+\sqrt{1-x^{2}}(1-y)\left(1-z^{2}\right)\right)^{2}-\left(1-R^{2}\right) .
\end{gathered}
$$

By $P_{\nu}^{(\alpha, \beta)}(x)(\nu=0,1, \ldots)$ we denote the Jacobi polynomials, i.e., the algebraic polynomials of degree $\nu$, orthogonal with the weight function $(1-x)^{\alpha}(1+x)^{\beta}$ on the segment $[-1,1]$, and normed by the condition

$$
P_{\nu}^{(\alpha, \beta)}(1)=1 \quad(\nu=0,1, \ldots) .
$$

Denote by $a_{n}(f)$ the Fourier-Jacobi coefficients of a function $f$, integrable with the weight function $\left(1-x^{2}\right)^{2}$ on the segment $[-1,1]$, with respect to the system of Jacobi polynomials $\left\{P_{n}^{(2,2)}(x)\right\}_{n=0}^{\infty}$, i.e., let

$$
a_{n}(f)=\int_{-1}^{1} f(x) P_{n}^{(2,2)}(x)\left(1-x^{2}\right)^{2} d x \quad(n=0,1, \ldots) .
$$

The following symmetric operator of generalised translation will play an auxiliary role in the sequel:

$$
T_{2 ; y}(f, x)=\frac{8}{3 \pi} \int_{-1}^{1}\left(1-z^{2}\right)^{2} f(R) \frac{d z}{\sqrt{1-z^{2}}},
$$

where

$$
R=x y-z \sqrt{1-x^{2}} \sqrt{1-y^{2}}
$$


2.

Lemma 2.1. The operator $\tau_{y}(f, x)$ has the following properties:

1) it is linear,

2) $\tau_{1}(f, x)=f(x)$,

3) $\tau_{y}\left(P_{\nu}^{(2,2)}, x\right)=P_{\nu}^{(2,2)}(x) P_{\nu}^{(0,4)}(y) \quad(\nu=0,1, \ldots)$,

4) $\tau_{y}(1, x)=1$,

5) $a_{n}\left(\tau_{y}(f, x)\right)=a_{n}(f) P_{n}^{(0,4)}(y) \quad(n=0,1, \ldots)$.

Proof. Properties 1) and 2) follow immediately from the definition of the operator $\tau_{y}(f, x)$.

In order to prove 3 , we consider the functions

$$
\begin{aligned}
& P_{m n}^{l}(z)= \\
& \quad P_{\nu}^{(\alpha, \beta)}(z)\left(\begin{array}{c}
\nu+\alpha \\
\alpha
\end{array}\right) 2^{-m} i^{m-n} \sqrt{\frac{(l-m) !(l+m) !}{(l-n) !(l+n) !}}(1-z)^{(m-n) / 2}(1+z)^{(m+n) / 2},
\end{aligned}
$$

where

$$
l=\nu+\frac{\alpha+\beta}{2}, \quad m=\frac{\alpha+\beta}{2}, \quad n=\frac{\alpha-\beta}{2} .
$$

Putting $n=0, m=k=2$ in the formula of multiplication for functions $P_{m n}^{l}$ (see [9, p. 138], we obtain the required equalities.

Property 4) is proved by means of $P_{0}^{(2,2)}(x)$ in 3$)$

We prove the equality in 5). To this effect, consider

$$
\begin{aligned}
I=a_{n}\left(\tau_{y}(f, x)\right) & =\int_{-1}^{1} \tau_{y}(f, x) P_{n}^{(2,2)}(x)\left(1-x^{2}\right)^{2} d x= \\
& =\frac{4}{\pi(1+y)^{2}} \int_{-1}^{1} \int_{-1}^{1} B_{y}(x, z, R) f(R) P_{n}^{(2,2)}(x)\left(1-x^{2}\right) \frac{d z d x}{\sqrt{1-z^{2}}},
\end{aligned}
$$

where

$$
\begin{gathered}
R=x y-z \sqrt{1-x^{2}} \sqrt{1-y^{2}} \\
B_{y}(x, z, R)=2\left(\sqrt{1-x^{2}} y+z x \sqrt{1-y^{2}}+\sqrt{1-x^{2}}(1-y)\left(1-z^{2}\right)\right)^{2}-\left(1-R^{2}\right) .
\end{gathered}
$$

Performing the change of variables

$$
\begin{gathered}
x=R y+V \sqrt{1-R^{2}} \sqrt{1-y^{2}} \\
z=-\frac{R \sqrt{1-y^{2}}-V y \sqrt{1-R^{2}}}{\sqrt{1-\left(R y+V \sqrt{1-R^{2}} \sqrt{1-y^{2}}\right)^{2}}}
\end{gathered}
$$

in the double integral, we obtain

$$
I=\frac{4}{\pi(1+y)^{2}} \int_{-1}^{1} \int_{-1}^{1} B_{y}(R, V, x)\left(1-R^{2}\right) f(R) P_{n}^{(2,2)}(x) \frac{d V d R}{\sqrt{1-V^{2}}} .
$$

Therefore,

$$
\begin{aligned}
I=\int_{-1}^{1} f(R)\left(1-R^{2}\right)^{2} & \frac{4}{\pi\left(1-R^{2}\right)(1+y)^{2}} \int_{-1}^{1} B_{y}(R, V, x) P_{n}^{(2,2)}(x) \\
& \times \frac{d V}{\sqrt{1-V^{2}}} d R=\int_{-1}^{1} f(R)\left(1-R^{2}\right)^{2} \tau_{y}\left(P_{n}^{(2,2)}, R\right) d R .
\end{aligned}
$$


Hence property 3) yields

$$
I=P_{n}^{(0,4)}(y) \int_{-1}^{1} f(R) P_{n}^{(2,2)}(R)\left(1-R^{2}\right)^{2} d R=a_{n}(f) P_{n}^{(0,4)}(y) .
$$

Lema 2.1 is proved.

Lemma 2.2. Let the numbers $p$ and $\alpha$ be such that $1 \leq p \leq \infty$;

$$
\begin{array}{rlrl}
1 / 2 & <\alpha \leq 1 & \text { for } p=1, \\
1-\frac{1}{2 p} & <\alpha<\frac{3}{2}-\frac{1}{2 p} & \text { for } 1<p<\infty, \\
1 & \leq \alpha<3 / 2 & \text { for } p=\infty . \\
R & =x \cos t-z \sqrt{1-x^{2}} \sin t .
\end{array}
$$

Then for every function $f \in L_{p, \alpha}$, we have

$$
\left\|\frac{1}{1-x^{2}} \int_{-1}^{1}\left(1-R^{2}\right)|f(R)| \frac{d z}{\sqrt{1-z^{2}}}\right\|_{p, \alpha} \leq C\|f\|_{p, \alpha}
$$

where the constant $C$ does not depend on $f$ and $x$.

Lemma 2.2 is proved in a more generalised form in [7].

Lemma 2.3. Let the numbers $p$ and $\alpha$ be such that $1 \leq p \leq \infty$;

$$
\begin{aligned}
& 1 / 2<\alpha \leq 1 \quad \text { for } p=1, \\
& 1-\frac{1}{2 p}<\alpha<\frac{3}{2}-\frac{1}{2 p} \text { for } 1<p<\infty \text {, } \\
& 1 \leq \alpha<3 / 2 \quad \text { for } p=\infty \text {. }
\end{aligned}
$$

If $f \in L_{p, \alpha}$, then

$$
\left\|\hat{\tau}_{t}(f, x)\right\|_{p, \alpha} \leq \frac{C}{\cos ^{4} t / 2}\|f\|_{p, \alpha},
$$

where constant $C$ does not depend on $f$ and $t$.

Proof. Let

$$
I=\left\|\hat{\tau}_{t}(f, x)\right\|_{p, \alpha}=\frac{1}{\pi \cos ^{4} t / 2}\left\|\frac{1}{1-x^{2}} \int_{-1}^{1} B_{\cos t}(x, z, R) f(R) \frac{d z}{\sqrt{1-z^{2}}}\right\|_{p, \alpha},
$$

where

$$
\begin{gathered}
R=x \cos t-z \sqrt{1-x^{2}} \sin t \\
B_{y}(x, z, R)=2\left(\sqrt{1+x^{2}} y+z x \sqrt{1-y^{2}}+\sqrt{1-x^{2}}(1-y)\left(1-z^{2}\right)\right)^{2}-\left(1-R^{2}\right) .
\end{gathered}
$$

Since

we have

$$
R^{2}+\left(\sqrt{1-x^{2}} y+z x \sqrt{1-y^{2}}\right)^{2}=1-\left(1-y^{2}\right)\left(1-z^{2}\right)
$$

$$
\left|\sqrt{1-x^{2}} y+z x \sqrt{1-y^{2}}\right| \leq \sqrt{1-R^{2}}
$$

and

$$
\left(1-y^{2}\right)\left(1-z^{2}\right) \leq 1-R^{2}
$$

Since $R$ is symmetric in $x$ and $y$, the last inequality yields

$$
\left(1-x^{2}\right)\left(1-z^{2}\right) \leq 1-R^{2} .
$$

Applying this inequality and inequality (2.2), we get

$$
\left|B_{y}(x, z, R)\right| \leq 19\left(1-R^{2}\right) .
$$


Applying Lemma 2.2, we obtain

$$
I \leq \frac{C_{1}}{\cos ^{4} t / 2}\left\|\frac{1}{1-x^{2}} \int_{-1}^{1}\left(1-R^{2}\right)|f(R)| \frac{d z}{\sqrt{1-z^{2}}}\right\|_{p, \alpha} \leq \frac{C_{2}}{\cos ^{4} t / 2}\|f\|_{p, \alpha} .
$$

Lemma 2.3 is proved.

Lemma 2.4. If $g(x) \tau_{y}(f, x) \in L_{1,2}$ for each $y \in(-1,1)$, then

$$
\int_{-1}^{1} f(x) \tau_{y}(g, x)\left(1-x^{2}\right)^{2} d x=\int_{-1}^{1} g(x) \tau_{y}(f, x)\left(1-x^{2}\right)^{2} d x .
$$

Proof. We have

$$
\begin{aligned}
I=\int_{-1}^{1} f(x) \tau_{y}(g, x) & \left(1-x^{2}\right)^{2} d x \\
= & \frac{4}{\pi(1+y)^{2}} \int_{-1}^{1} \int_{-1}^{1} f(x) g(R) B_{y}(x, z, R)\left(1-x^{2}\right) \frac{d z d x}{\sqrt{1-z^{2}}},
\end{aligned}
$$

where

$$
\begin{gathered}
R=x \cos t-z \sqrt{1-x^{2}} \sin t \\
B_{y}(x, z, R)=2\left(\sqrt{1-x^{2}} y+z x \sqrt{1-y^{2}}+\sqrt{1-x^{2}}(1-y)\left(1-z^{2}\right)\right)^{2}-\left(1-R^{2}\right) .
\end{gathered}
$$

Performing the change of variables in this double integral by formulas (2.1), we obtain

$$
\begin{aligned}
I=\frac{4}{\pi(1+y)^{2}} \int_{-1}^{1} \int_{-1}^{1} f(x) g(R) B_{y}(R, V, x) & \left(1-R^{2}\right) \frac{d V d R}{\sqrt{1-V^{2}}} \\
& =\int_{-1}^{1} g(R) \tau_{y}(f, R)\left(1-R^{2}\right)^{2} d R .
\end{aligned}
$$

Lemma 2.4 is proved.

Lemma 2.5. Assume that the derivative $f^{\prime}(x)$ is absolutely continuous on every segment $[a, b] \subset(-1,1)$ and $D_{x, 2,2} f(x) \in L_{1,2}$. Then

1) for fixed $y \in(-1,1)$, the derivative $\frac{d}{d x} \tau_{y}(f, x)$ is absolutely continuous on every segment $[c, d] \subset(-1,1)$,

2) for almost every $x \in(-1,1)$ and every $y \in(-1,1)$, the following equality holds true

$$
\tau_{y}\left(D_{x, 2,2} f, x\right)=D_{x, 2,2} \tau_{y}(f, x) .
$$

Proof. In order to prove 1), we consider the function

$$
\varphi(x)=\frac{B_{y}(x, z, R)}{\left(1-x^{2}\right)(1+y)^{2} \sqrt{1-z^{2}}} f(R),
$$

where $B_{y}(x, z, R)$ and $R$ have been defined in Lemma 2.4. It is obvious that the function $\varphi^{\prime}(x)$ is continuous on every segment $[c, d] \subset(-1,1)$. Hence 1$)$ follows by applying Lebesgue's dominated convergence theorem.

In order to prove 2) first we prove the equality

$$
\tau_{y}\left(D_{x, 2,2} f, x\right)=D_{x, 2,2} \tau_{y}(f, x)
$$

for infinitely differentiable functions $f$ which are equal to zero outside of some segment $[a, b] \subset(-1,-y) \cup(-y, y) \cup(y, 1)$.

From 1) it follows that $D_{x, 2,2} \tau_{y}(f, x)$ exists. 
Assume that the function $f$ is infinitely differentiable and is equal to zero outside of some segment $[a, b] \subset(-1,-y) \cup(-y, y) \cup(y, 1)$. Applying Lemmas 2.4 and 2.1] we obtain

$$
\begin{aligned}
I=\int_{-1}^{1} \tau_{y}\left(D_{x, 2,2} f, x\right) P_{n}^{(2,2)}(x) & \left(1-x^{2}\right)^{2} d x \\
= & P_{n}^{(0,4)}(y) \int_{-1}^{1} D_{x, 2,2} f(x) P_{n}^{(2,2)}(x)\left(1-x^{2}\right)^{2} d x .
\end{aligned}
$$

Integrating by parts twice and taking into account that $f(x)=0$ and $f^{\prime}(x)=0$ outside of $[a, b] \subset(-1,1)$, we have

$$
I=P_{n}^{(0,4)}(y) \int_{-1}^{1} D_{x, 2,2} P_{n}^{(2,2)}(x) f(x)\left(1-x^{2}\right)^{2} d x .
$$

It is well known [2, p. 171] that

$$
D_{x, 2,2} P_{n}^{(2,2)}(x)=-n(n+5) P_{n}^{(2,2)}(x) .
$$

Therefore

$$
I=-n(n+5) P_{n}^{(0,4)}(y) \int_{-1}^{1} f(x) P_{n}^{(2,2)}(x)\left(1-x^{2}\right)^{2} d x .
$$

Applying Lemmas 2.1 and 2.4, integrating by parts twice, and considering that $\tau_{y}(f, x)=0$ outside of some segment $[\gamma, \delta] \subset(-1,1)$, we obtain

$$
I=\int_{-1}^{1} D_{x, 2,2} \tau_{y}(f, x) P_{n}^{(2,2)}(x)\left(1-x^{2}\right)^{2} d x .
$$

Thus for fixed $y$, all the Fourier-Jacobi coefficients of the function

$$
F(x)=\tau_{y}\left(D_{x, 2,2} f, x\right)-D_{x, 2,2} \tau_{y}(f, x)
$$

with respect to the system $\left\{P_{n}^{(2,2)}(x)\right\}_{n=0}^{\infty}$ of polynomials are equal to zero. Hence it follows that $F(x)=0$ almost everywhere on $[-1,1]$.

Thus, equality (2.3) has been proved for infinitely differentiable functions which are equal to zero outside of some segment $[a, b] \subset(-1,-y) \cup(-y, y) \cup(y, 1)$.

Now, let the function $f(x)$ satisfy the conditions of the lemma. Let a function $g(x)$ be infinitely differentiable and equal to zero outside of some segment $[c, d] \subset(-1,-y) \cup(-y, y) \cup(y, 1)$. Integrating by parts twice and taking into account that

$$
\begin{gathered}
g(x)\left(1-x^{2}\right)^{3} \frac{d}{d x} \tau_{y}(f, x) \rightarrow 0 \quad \text { and } \quad \tau_{y}(f, x)\left(1-x^{2}\right)^{3} \frac{d}{d x} g(x) \rightarrow 0 \\
\text { for } x \rightarrow-1+0 \quad \text { and } \quad x \rightarrow 1-0
\end{gathered}
$$

we obtain

$$
J_{1}=\int_{-1}^{1} D_{x, 2,2} \tau_{y}(f, x) g(x)\left(1-x^{2}\right)^{2} d x=\int_{-1}^{1} D_{x, 2,2} g(x) \tau_{y}(f, x)\left(1-x^{2}\right)^{2} d x .
$$

Applying Lemma 2.1 we get

$$
J_{1}=\int_{-1}^{1} f(x) \tau_{y}\left(D_{x, 2,2} g, x\right)\left(1-x^{2}\right)^{2} d x .
$$

On the other hand, let

$$
J_{2}=\int_{-1}^{1} \tau_{y}\left(D_{x, 2,2} f, x\right) g(x)\left(1-x^{2}\right)^{2} d x .
$$


Applying Lemma 2.4 and then integrating by parts twice, we have

$$
J_{2}=\int_{-1}^{1} D_{x, 2,2} \tau_{y}(g, x) f(x)\left(1-x^{2}\right)^{2} d x
$$

Therefore, we obtain

$$
J_{2}-J_{1}=\int_{-1}^{1}\left(D_{x, 2,2} \tau_{y}(g, x)-\tau_{y}\left(D_{x, 2,2} g, x\right)\right) f(x)\left(1-x^{2}\right)^{2} d x .
$$

But for the function $g(x)$ we have already proved equality (2.3) for almost every $x \in(-1,1)$. Hence

$$
J_{2}-J_{1}=\int_{-1}^{1}\left(\tau_{y}\left(D_{x, 2,2} f, x\right)-D_{x, 2,2} \tau_{y}(f, x)\right) g(x)\left(1-x^{2}\right)^{2} d x=0
$$

for every $y$. Now, equality (2.3) follows from the fact that the segment $[c, d] \subset$ $(-1,-y) \cup(-y, y) \cup(y, 1)$ and the function $g(x)$ can be arbitrarily chosen.

Lemma 2.5 is proved.

Lemma 2.6. Assume that the derivative $f^{\prime}(x)$ is absolutely continuous on every segment $[a, b] \subset(-1,1)$ and $D_{x, 2,2} f(x) \in L_{1,2}$. Then for almost every $x \in(-1,1)$ and every $y \in(-1,1)$

$$
\tau_{y}(f, x)-f(x)=\int_{1}^{y}(1-v)^{-1}(1+v)^{-5} \int_{1}^{v}(1+u)^{4} \tau_{u}\left(D_{x, 2,2} f, x\right) d u d v
$$

and

$$
\begin{aligned}
\tau_{y}(f, x)-\tau_{0} & (f, x) \\
= & -\int_{0}^{y}(1-v)^{-1}(1+v)^{-5} \int_{v}^{-1}(1+u)^{4} \tau_{u}\left(D_{x, 2,2} f, x\right) d u d v .
\end{aligned}
$$

Proof. We prove equality (2.4). If $f$ is an infinitely differentiable function, equal to zero outside of some segment $[a, b] \subset(-1,-y) \cup(-y, y) \cup(y, 1)$, then for almost every $x \in(-1,1)$ and almost every $u \in(-1,1)$ the following equality holds true

$$
\tau_{u}\left(D_{x, 2,2} f, x\right)=D_{u, 0,4} \tau_{u}(f, x) .
$$

Applying this equality and Lemma 2.1, we obtain

$$
\begin{aligned}
& \int_{1}^{y}(1-v)^{-1}(1+v)^{-5} \int_{1}^{v}(1+u)^{4} \tau_{u}\left(D_{x, 2,2} f, x\right) d u d v \\
& \quad=\int_{1}^{y}(1-v)^{-1}(1+v)^{-5} \int_{1}^{v}(1+u)^{4} D_{u, 0,4} \tau_{u}(f, x) d u d v=\tau_{y}(f, x)-f(x) .
\end{aligned}
$$

Now let the function $f(x)$ satisfy the conditions of the lemma and let $g(x)$ be an infinitely differentiable function, equal to zero outside of some segment $[c, d] \subset(-1,-y) \cup(-y, y) \cup(y, 1)$. Then by Lemma 2.4. analogously to the proof of Lemma 2.5, while integrating by parts twice, it is easy to prove that

$$
\begin{aligned}
& J=\int_{-1}^{1} \int_{1}^{y}(1-v)^{-1}(1+v)^{-5} \int_{1}^{v}(1+u)^{4} \tau_{u}\left(D_{x, 2,2} f, x\right) g(x)\left(1-x^{2}\right)^{2} d u d v d x \\
& =\int_{-1}^{1} f(x)\left(1-x^{2}\right)^{2} \int_{1}^{y}(1-v)^{-1}(1+v)^{-5} \int_{1}^{v}(1+u)^{4} D_{x, 2,2} \tau_{u}(g, x) d u d v d x .
\end{aligned}
$$

Making use of Lemma 2.5 and the fact that we have already proved equality (2.4 for almost every $x \in(-1,1)$ in the case of any infinitely differentiable function $g(x)$, equal to zero outside of the segment $[c, d] \subset(-1,-y) \cup(-y, y) \cup(y, 1)$, we obtain

$$
J=\int_{-1}^{1}\left(\tau_{y}(g, x)-g(x)\right) f(x)\left(1-x^{2}\right)^{2} d x .
$$


Applying once more Lemma 2.4, we get that

$$
J=\int_{-1}^{1}\left(\tau_{y}(f, x)-f(x)\right) g(x)\left(1-x^{2}\right)^{2} d x .
$$

Hence equality (2.4) follows by taking into account the fact that the segment $[c, d]$ and the function $g(x)$ can be arbitrarily chosen.

Equality (2.5) is proved in an analogous way.

Lemma 2.6 is proved.

Corollary 2.1. Assume that the derivative $f^{\prime}(x)$ is absolutely continuous on every segment $[a, b] \subset(-1,1)$ and $D_{x, 2,2} f(x) \in L_{1,2}$. Then for almost every $x \in(-1,1)$ and every $t \in(-\pi, \pi)$

$$
\begin{aligned}
\hat{\tau}_{t}(f, x)- & f(x) \\
& =\int_{0}^{t}(\sin v / 2)^{-1}(\cos v / 2)^{-9} \int_{0}^{v} \hat{\tau}_{u}\left(D_{x, 2,2} f, x\right) \sin u / 2(\cos u / 2)^{9} d u d v
\end{aligned}
$$

and

$$
\begin{aligned}
\hat{\tau}_{t}(f, x) & -\hat{\tau}_{\pi / 2}(f, x) \\
= & -\int_{\pi / 2}^{t}(\sin v / 2)^{-1}(\cos v / 2)^{-9} \int_{v}^{\pi} \hat{\tau}_{u}\left(D_{x, 2,2} f, x\right) \sin u / 2(\cos u / 2)^{9} d u d v .
\end{aligned}
$$

The first equality follows immediately from equality (2.4) by substituting $\cos u$ and $\cos v$ for $u$ and $v$, respectively. In an analogous way, the second equality follows from equality (2.5).

Lemma 2.7. Let $P_{n}$ be an algebraic polynomial of degree not greater than $n-1$, $1 \leq p \leq \infty, \rho \geq 0$

$$
\begin{array}{ll}
\alpha>-1 / p & \text { for } 1 \leq p<\infty, \\
\alpha \geq 0 & \text { for } p=\infty .
\end{array}
$$

Then the following inequalities hold true:

$$
\begin{gathered}
\left\|P_{n}^{\prime}\right\|_{p, \alpha+1 / 2} \leq C_{1} n\left\|P_{n}\right\|_{p, \alpha}, \\
\left\|P_{n}\right\|_{p, \alpha} \leq C_{2} n^{2 \rho}\left\|P_{n}\right\|_{p, \alpha+\rho},
\end{gathered}
$$

where the constants $C_{1}$ and $C_{2}$ do not depend on $n$.

Lemma is proved in 4 .

Lemma 2.8. Let $q$ and $m$ be natural numbers and let $f \in L_{1,2}$. Then the function

$$
Q(x)=\int_{0}^{\pi} T_{2 ; \cos t}(f, x)\left(\frac{\sin \frac{m t}{2}}{\sin \frac{t}{2}}\right)^{2 q+4} \sin ^{5} t d t
$$

is an algebraic polynomial of degree not greater than $(q+2)(m-1)$.

Lemma is proved in [5].

Lemma 2.9. Let the numbers $p$ and $\alpha$ be such that $1 \leq p \leq \infty$;

$$
\begin{aligned}
-1 / 2 & <\alpha \leq 2 & & \text { for } p=1, \\
-\frac{1}{2 p} & <\alpha<5 / 2-\frac{1}{2 p} & & \text { for } 1<p<\infty, \\
0 & \leq \alpha<5 / 2 & & \text { for } p=\infty .
\end{aligned}
$$

If $f \in A D(p, \alpha)$, then

$$
E_{n}(f)_{p, \alpha} \leq C \frac{1}{n^{2}}\left\|D_{x, 2,2} f(x)\right\|_{p, \alpha},
$$


where the constant $C$ does not depend on $f$ and $n$.

Proof. For a fixed natural number $q>2$, we chose the natural number $m$ such that

$$
\frac{n-1}{q+2}<m \leq \frac{n-1}{q+2}+1 \text {. }
$$

It is easy to prove that under the conditions of the lemma, $f \in L_{p, \alpha}$ implies $f \in L_{1,2}$. Hence by Lemma 2.8, it follows that the function

$$
Q(x)=\frac{1}{\gamma_{m}} \int_{0}^{\pi} T_{2 ; \cos t}(f, x)\left(\frac{\sin \frac{m t}{2}}{\sin \frac{t}{2}}\right)^{2 q+4} \sin ^{5} t d t,
$$

where

$$
\gamma_{m}=\int_{0}^{\pi}\left(\frac{\sin \frac{m t}{2}}{\sin \frac{t}{2}}\right)^{2 q+4} \sin ^{5} t d t
$$

is an algebraic polynomial of degree not greater than $n-1$. Therefore, by applying the generalised Minkowski inequality, we have

$$
\begin{aligned}
& E_{n}(f)_{p, \alpha} \leq\|f-Q\|_{p, \alpha} \\
& \quad \leq \frac{1}{\gamma_{m}} \int_{0}^{\pi}\left\|T_{2 ; \cos t}(f, x)-f(x)\right\|_{p, \alpha}\left(\frac{\sin \frac{m t}{2}}{\sin \frac{t}{2}}\right)^{2 q+4} \sin ^{5} t d t .
\end{aligned}
$$

Reasoning as in the proof of inequality (2.2) of Theorem 3.1, i.e., applying the appropriately modified versions of Lemmas 2.6 and 2.3 for the operator $T_{2 ; \cos t}(f, x)$, we obtain

$$
E_{n}(f)_{p, \alpha} \leq C_{1}\left\|D_{x, 2,2} f(x)\right\|_{p, \alpha} \frac{1}{\gamma_{m}} \int_{0}^{\pi} t^{2}\left(\frac{\sin \frac{m t}{2}}{\sin \frac{t}{2}}\right)^{2 q+4} \sin ^{5} t d t .
$$

Making use of an estimate of Jackson kernel, we get

$$
E_{n}(f)_{p, \alpha} \leq C_{2} \frac{1}{m^{2}}\left\|D_{x, 2,2} f(x)\right\|_{p, \alpha} \leq C_{3} \frac{1}{n^{2}}\left\|D_{x, 2,2} f(x)\right\|_{p, \alpha} .
$$

Lema 2.9 is proved.

3.

Theorem 3.1. Let the numbers $p$ and $\alpha$ be such that $1 \leq p \leq \infty$;

$$
\begin{aligned}
1 / 2 & <\alpha \leq 1 & & \text { for } p=1, \\
1-\frac{1}{2 p} & <\alpha<\frac{3}{2}-\frac{1}{2 p} & & \text { for } 1<p<\infty, \\
1 & \leq \alpha<3 / 2 & & \text { for } p=\infty .
\end{aligned}
$$

If $f \in L_{p, \alpha}$, then for all $\delta \in[0, \pi)$,

$$
C_{1} K(f, \delta)_{p, \alpha} \leq \hat{\omega}(f, \delta)_{p, \alpha} \leq C_{2} \frac{1}{\cos ^{4} \delta / 2} K(f, \delta)_{p, \alpha}
$$

where the positive constants $C_{1}$ and $C_{2}$ do not depend on $f$ and $\delta$.

Proof. We prove that for every function $g(x) \in A D(p, \alpha)$ and every $t \in(-\pi, \pi)$, we have

$$
\left\|\hat{\tau}_{t}(g, x)-g(x)\right\|_{p, \alpha} \leq C_{3} \frac{1}{\cos ^{4} t / 2} t^{2}\left\|D_{x, 2,2} g(x)\right\|_{p, \alpha},
$$

where the constant $C_{3}$ does not depend on $g$ dhe $t$. 
Let $0<t \leq \pi / 2$. Then Corollary of Lemma 2.6 yields

$$
\begin{aligned}
I_{1} & =\left\|\hat{\tau}_{t}(g, x)-g(x)\right\|_{p, \alpha} \\
& =\left\|\int_{0}^{t}(\sin v / 2)^{-1}(\cos v / 2)^{-9} \int_{0}^{v} \hat{\tau}_{u}\left(D_{x, 2,2} g, x\right) \sin u / 2(\cos u / 2)^{9} d u d v\right\|_{p, \alpha} .
\end{aligned}
$$

Applying the generalised Minkowski inequality and Lemma 2.3, we get

$$
\begin{aligned}
I_{1} \leq & \int_{0}^{t}(\sin v / 2)^{-1}(\cos v / 2)^{-9} \\
& \times \int_{0}^{v}\left\|\hat{\tau}_{u}\left(D_{x, 2,2} g, x\right)\right\|_{p, \alpha} \sin u / 2(\cos u / 2)^{9} d u d v \\
\leq & C_{4}\left\|D_{x, 2,2} g(x)\right\|_{p, \alpha} \int_{0}^{t}(\sin v / 2)^{-1}(\cos v / 2)^{-9} \int_{0}^{v}\left(\sin \frac{u}{2}\right)\left(\cos \frac{u}{2}\right)^{5} d u d v .
\end{aligned}
$$

Since the inequality

$$
\int_{0}^{t}(\sin v / 2)^{-1}(\cos v / 2)^{-9} \int_{0}^{v}\left(\sin \frac{u}{2}\right)\left(\cos \frac{u}{2}\right)^{5} d u d v \leq C_{5} t^{2}
$$

holds for $0<t \leq \pi / 2$, we obtain

$$
I_{1} \leq C_{6} t^{2}\left\|D_{x, 2,2} g(x)\right\|_{p, \alpha} \leq C_{6} \frac{1}{\cos ^{4} t / 2} t^{2}\left\|D_{x, 2,2} g(x)\right\|_{p, \alpha} .
$$

For $t=0$ inequality (3.1) is trivial.

Let $\pi / 2 \leq t<\pi$. Then by Corollary of Lemma 2.6. we get

$$
\begin{aligned}
I_{2} & =\left\|\hat{\tau}_{t}(g, x)-\hat{\tau}_{\pi / 2}(g, x)\right\|_{p, \alpha} \\
& =\left\|\int_{\pi / 2}^{t}(\sin v / 2)^{-1}(\cos v / 2)^{-9} \int_{v}^{\pi} \hat{\tau}_{u}\left(D_{x, 2,2} g, x\right) \sin u / 2(\cos u / 2)^{9} d u d v\right\|_{p, \alpha} .
\end{aligned}
$$

Applying the generalised Minkowski inequality and then Lemma 2.3, we have

$$
\begin{aligned}
I_{2} \leq C_{7} \| D_{x, 2,2} g(x) & \|_{p, \alpha} \\
& \times \int_{\pi / 2}^{t}(\sin v / 2)^{-1}(\cos v / 2)^{-9} \int_{v}^{\pi}\left(\sin \frac{u}{2}\right)\left(\cos \frac{u}{2}\right)^{5} d u d v .
\end{aligned}
$$

Considering that for $\pi / 2 \leq t<\pi$ we have

$$
\int_{\pi / 2}^{t}(\sin v / 2)^{-1}(\cos v / 2)^{-9} \int_{v}^{\pi}\left(\sin \frac{u}{2}\right)\left(\cos \frac{u}{2}\right)^{5} d u d v \leq C_{8} \frac{1}{\cos ^{4} t / 2}
$$

it follows that

$$
I_{2} \leq C_{9} \frac{1}{\cos ^{4} t / 2}\left\|D_{x, 2,2} g(x)\right\|_{p, \alpha} \leq C_{9} \frac{1}{\cos ^{4} t / 2} t^{2}\left\|D_{x, 2,2} g(x)\right\|_{p, \alpha} .
$$

Since

$$
\left\|\hat{\tau}_{t}(g, x)-g(x)\right\|_{p, \alpha} \leq\left\|\hat{\tau}_{t}(g, x)-\hat{\tau}_{\pi / 2}(g, x)\right\|_{p, \alpha}+\left\|\hat{\tau}_{\pi / 2}(g, x)-g(x)\right\|_{p, \alpha},
$$

applying inequality (3.2) and the fact that inequality (3.1) has been proved for $0 \leq t \leq \pi / 2$, we obtain

$$
\left\|\hat{\tau}_{t}(g, x)-g(x)\right\|_{p, \alpha} \leq C_{10} \frac{1}{\cos ^{4} t / 2} t^{2}\left\|D_{x, 2,2} g(x)\right\|_{p, \alpha}
$$

for $\pi / 2 \leq t<\pi$.

Thus, inequality (3.1) is proved for $0 \leq t<\pi$. Since

$$
\tau_{\cos t}(g, x)=\tau_{\cos (-t)}(g, x),
$$


we conclude that inequality (3.1) holds for every $t \in(-\pi, \pi)$.

Let $f \in L_{p, \alpha}$ and $0 \leq|t| \leq \delta<\pi$. Then for every function $g(x) \in A D(p, \alpha)$, applying Lemma 2.3 gives

$$
\begin{aligned}
\left\|\hat{\tau}_{t}(f, x)-f(x)\right\|_{p, \alpha} \leq \| \hat{\tau}_{t}(f & -g, x)\left\|_{p, \alpha}+\right\| \hat{\tau}_{t}(g, x)-g(x)\left\|_{p, \alpha}+\right\| g-f \|_{p, \alpha} \\
& \leq C_{11} \frac{1}{\cos ^{4} t / 2}\|f-g\|_{p, \alpha}+\left\|\hat{\tau}_{t}(g, x)-g(x)\right\|_{p, \alpha} .
\end{aligned}
$$

Making use of inequality (3.1), we get

$$
\left\|\hat{\tau}_{t}(f, x)-f(x)\right\|_{p, \alpha} \leq C_{12} \frac{1}{\cos ^{4} t / 2}\left(\|f-g\|_{p, \alpha}+t^{2}\left\|D_{x, 2,2} g(x)\right\|_{p, \alpha}\right),
$$

where the constant $C_{12}$ does not depend on $f, g$ and $t$. This proves the right-hand side inequality of the theorem.

In order to prove the left-hand side inequality, we consider the function

$$
g_{\delta}(x)=\frac{1}{\kappa(\delta)} \int_{0}^{\delta}(\sin v / 2)^{-1}(\cos v / 2)^{-9} \int_{0}^{v} \hat{\tau}_{u}(f, x) \sin u / 2(\cos u / 2)^{9} d u d v,
$$

where

$$
\kappa(\delta)=\int_{0}^{\delta}(\sin v / 2)^{-1}(\cos v / 2)^{-9} \int_{0}^{v} \sin u / 2(\cos u / 2)^{9} d u d v .
$$

Let $0<\delta<\pi / 2$, then

$$
C_{13} \delta^{2} \leq \kappa(\delta) \leq C_{14} \delta^{2}
$$

Applying the generalised Minkowski inequality and Lemma 2.3. we obtain

$$
\begin{array}{r}
\left\|g_{\delta}(x)\right\|_{p, \alpha} \\
\leq \frac{1}{\kappa(\delta)} \int_{0}^{\delta}(\sin v / 2)^{-1}(\cos v / 2)^{-9} \int_{0}^{v}\left\|\hat{\tau}_{u}(f, x)\right\|_{p, \alpha} \sin u / 2(\cos u / 2)^{9} d u d v \\
\leq C_{15} \frac{1}{\cos ^{4} \delta / 2}\|f\|_{p, \alpha},
\end{array}
$$

that is, $g_{\delta}(x) \in L_{p, \alpha}$.

Put

where

$$
g(x)=-\int_{0}^{x}\left(1-y^{2}\right)^{-3} \int_{y}^{1}\left(1-z^{2}\right)^{2}\left(f(z)-\frac{c_{1}}{c_{0}}\right) d z d y,
$$

Since

$$
c_{1}=\int_{-1}^{1}\left(1-z^{2}\right)^{2} f(z) d z, \quad c_{0}=\int_{-1}^{1}\left(1-z^{2}\right)^{2} d z
$$

we have

$$
D_{x, 2,2} g(x)=f(x)-\frac{c_{1}}{c_{0}},
$$

$$
\begin{aligned}
g_{\delta}(x)=\frac{1}{\kappa(\delta)} \int_{0}^{\delta}(\sin v / 2)^{-1}( & \cos v / 2)^{-9} \\
& \times \int_{0}^{v} \hat{\tau}_{u}\left(D_{x, 2,2} g, x\right) \sin u / 2(\cos u / 2)^{9} d u d v+\frac{c_{1}}{c_{0}} .
\end{aligned}
$$

Applying Corollary of Lemma 2.6 gives

$$
g_{\delta}(x)=\frac{1}{\kappa(\delta)}\left(\hat{\tau}_{\delta}(g, x)-g(x)\right)+\frac{c_{1}}{c_{0}} .
$$

Applying the operator $D_{x, 2,2}$ and then Lemma 2.5, it follows that

$$
D_{x, 2,2} g_{\delta}(x)=\frac{1}{\kappa(\delta)}\left(\hat{\tau}_{\delta}\left(D_{x, 2,2} g, x\right)-D_{x, 2,2} g(x)\right)=\frac{1}{\kappa(\delta)}\left(\hat{\tau}_{\delta}(f, x)-f(x)\right) .
$$


Therefore, by Lemmas 2.3 and 2.5. we conclude that $g_{\delta}(x) \in A D(p, \alpha)$.

By the last equality and inequality (3.3), we obtain

$$
\left\|D_{x, 2,2} g_{\delta}(x)\right\|_{p, \alpha} \leq C_{16} \frac{1}{\delta^{2}}\left\|\hat{\tau}_{\delta}(f, x)-f(x)\right\|_{p, \alpha},
$$

that is,

$$
\left\|D_{x, 2,2} g_{\delta}(x)\right\|_{p, \alpha} \leq C_{16} \frac{1}{\delta^{2}} \hat{\omega}(f, \delta)_{p, \alpha} .
$$

On the other hand, by applying the Minkowsky inequality, we get

$$
\begin{aligned}
\left\|f(x)-g_{\delta}(x)\right\|_{p, \alpha} & \leq \frac{1}{\kappa(\delta)} \int_{0}^{\delta}(\sin v / 2)^{-1}(\cos v / 2)^{-9} \\
& \times \int_{0}^{v}\left\|f(x)-\hat{\tau}_{u}(f, x)\right\|_{p, \alpha} \sin u / 2(\cos u / 2)^{9} d u d v \leq \hat{\omega}(f, \delta)_{p, \alpha} .
\end{aligned}
$$

Thus, for $0<\delta \leq \pi / 2$ we have proved that

$$
I(\delta)=\left\|f(x)-g_{\delta}(x)\right\|_{p, \alpha}+\delta^{2}\left\|D_{x, 2,2} g_{\delta}(x)\right\|_{p, \alpha} \leq C_{17} \hat{\omega}(f, \delta)_{p, \alpha} .
$$

Since for $\pi / 2 \leq \delta<\pi$ we have $\delta^{2}<\pi^{2} \cdot 1$ and $1<\pi / 2$, it follows that

$$
\begin{aligned}
K(f, \delta)_{p, \alpha} \leq \pi^{2}\left(\left\|f(x)-g_{1}(x)\right\|_{p, \alpha}\right. & \left.+1^{2} \cdot\left\|D_{x, 2,2} g_{1}(x)\right\|_{p, \alpha}\right) \\
& =\pi^{2} I(1) \leq \pi^{2} C_{17} \hat{\omega}(f, 1)_{p, \alpha} \leq C_{18} \hat{\omega}(f, \delta)_{p, \alpha} .
\end{aligned}
$$

Thus, we have proved the left-hand side inequality of the theorem for $0<\delta<\pi$. For $\delta=0$ this inequality is trivial.

Theorem 3.1 is proved.

Theorem 3.2. Let the numbers $p$ and $\alpha$ be such that $1 \leq p \leq \infty$;

$$
\begin{aligned}
1 / 2 & <\alpha \leq 1 & & \text { for } p=1, \\
1-\frac{1}{2 p} & <\alpha<\frac{3}{2}-\frac{1}{2 p} & & \text { for } 1<p<\infty, \\
1 & \leq \alpha<3 / 2 & & \text { for } p=\infty .
\end{aligned}
$$

If $f \in L_{p, \alpha}$, then for every natural number $n$

$$
C_{1} E_{n}(f)_{p, \alpha} \leq \hat{\omega}(f, 1 / n)_{p, \alpha} \leq C_{2} \frac{1}{n^{2}} \sum_{\nu=1}^{n} \nu E_{\nu}(f)_{p, \alpha},
$$

where the positive constants $C_{1}$ and $C_{2}$ do not depend on $f$ and $n$.

Proof. For every function $g(x) \in A D(p, \alpha)$, we have

$$
E_{n}(f)_{p, \alpha} \leq E_{n}(f-g)_{p, \alpha}+E_{n}(g)_{p, \alpha} .
$$

Applying Lemma 2.9 gives

$$
E_{n}(f)_{p, \alpha} \leq\|f-g\|_{p, \alpha}+C_{3} \frac{1}{n^{2}}\left\|D_{x, 2,2} g(x)\right\|_{p, \alpha},
$$

where the constant $C_{3}$ does not depend on $f, g$ and $n$. Therefore, we get

$$
E_{n}(f)_{p, \alpha} \leq C_{4} K(f, 1 / n)_{p, \alpha} .
$$

Hence Theorem 3.1 yields

$$
E_{n}(f)_{p, \alpha} \leq C_{5} \hat{\omega}(f, 1 / n)_{p, \alpha},
$$

which proves the left-hand side inequality of the theorem. 
We prove the right-hand side inequality. Let $P_{n}(x)$ be the algebraic polynomial of best approximation for $f$ in the metrics $L_{p, \alpha}$ whose degree is not greater than $n-1$. Let $k$ be chosen such that

$$
n / 2<2^{k} \leq n+1
$$

Since $P_{2^{k}}(x) \in A D(p, \alpha)$, Theorem 3.1 yields

$$
\begin{aligned}
\hat{\omega}(f, 1 / n)_{p, \alpha} \leq C_{6} \frac{1}{\left(\cos \frac{1}{2 n}\right)^{4}} K(f, 1 / n)_{p, \alpha} & \\
& \leq C_{7}\left(\left\|f-P_{2^{k}}\right\|_{p, \alpha}+\frac{1}{n^{2}}\left\|D_{x, 2,2} P_{2^{k}}\right\|_{p, \alpha}\right) .
\end{aligned}
$$

Since

$$
D_{x, 2,2} P_{2^{k}}(x)=\sum_{\nu=0}^{k-1} D_{x, 2,2}\left(P_{2^{\nu+1}}(x)-P_{2^{\nu}}(x)\right),
$$

Lemma 2.7 yields

$$
\begin{aligned}
\left\|D_{x, 2,2} P_{n}(x)\right\|_{p, \alpha} \leq\left\|\left(1-x^{2}\right) P_{n}^{\prime \prime}(x)\right\|_{p, \alpha} & +6\left\|P_{n}^{\prime}(x)\right\|_{p, \alpha} \\
& \leq C_{8} n\left\|P_{n}^{\prime}(x)\right\|_{p, \alpha+1 / 2} \leq C_{9} n^{2}\left\|P_{n}\right\|_{p, \alpha},
\end{aligned}
$$

whence we obtain

$$
\begin{gathered}
\left\|D_{x, 2,2} P_{2^{k}}(x)\right\|_{p, \alpha} \leq C_{10} \sum_{\nu=0}^{k-1} 2^{2(\nu+1)}\left\|P_{2^{\nu+1}}(x)-P_{2^{\nu}}(x)\right\|_{p, \alpha} \\
\leq C_{10} \sum_{\nu=0}^{k-1} 2^{2(\nu+1)}\left(\left\|P_{2^{\nu+1}}(x)-f(x)\right\|_{p, \alpha}+\left\|f(x)-P_{2^{\nu}}(x)\right\|_{p, \alpha}\right) \\
\leq C_{10} \sum_{\nu=0}^{k-1} 2^{2(\nu+1)}\left(E_{2^{\nu+1}}(f)_{p, \alpha}+E_{2^{\nu}}(f)_{p, \alpha}\right) \leq 2 C_{10} \sum_{\nu=0}^{k-1} 2^{2(\nu+1)} E_{2^{\nu}}(f)_{p, \alpha} .
\end{gathered}
$$

Therefore, inequality (3.4) implies

$$
\begin{aligned}
\hat{\omega}(f, 1 / n)_{p, \alpha} \leq C_{11}\left(E_{2^{k}}(f)_{p, \alpha}+\frac{1}{n^{2}} \sum_{\nu=0}^{k-1} 2^{2(\nu+1)} E_{2^{\nu}}(f)_{p, \alpha}\right) & \\
& \leq C_{11} \frac{1}{n^{2}} \sum_{\nu=0}^{k} 2^{2(\nu+1)} E_{2^{\nu}}(f)_{p, \alpha} .
\end{aligned}
$$

We note that

$$
\sum_{\mu=2^{\nu-1}}^{2^{\nu}-1} \mu E_{\mu}(f)_{p, \alpha} \geq 2^{2(\nu-1)} E_{2^{\nu}}(f)_{p, \alpha}
$$

holds for $\nu=1,2, \ldots, k$. Hence we have

$$
\begin{aligned}
& \hat{\omega}(f, 1 / n)_{p, \alpha} \leq C_{12} \frac{1}{n^{2}}\left(4 E_{1}\right.\left.(f)_{p, \alpha}+\sum_{\nu=1}^{k} \sum_{\mu=2^{\nu-1}}^{2^{\nu}-1} \mu E_{\mu}(f)_{p, \alpha}\right) \\
& \leq C_{13} \frac{1}{n^{2}} \sum_{\nu=1}^{2^{k}-1} \nu E_{\nu}(f)_{p, \alpha} \leq C_{13} \frac{1}{n^{2}} \sum_{\nu=1}^{n} \nu E_{\nu}(f)_{p, \alpha} .
\end{aligned}
$$

Theorem 3.2 is proved. 


\section{REFERENCES}

1. P. L. Butzer, R. L. Stens, and M. Wehrens, Higher order moduli of continuity based on the Jacobi translation operator and best approximation, C. R. Math. Rep. Acad. Sci. Canada 2 (1980), no. 2, 83-88. MR 81a:41040

2. A. Erdélyi, W. Magnus, F. Oberhettinger, and F. G. Tricomi, Higher transcendental functions, Three volumes, Robert E. Krieger Publishing Co. Inc., Melbourne, Fla., 1981, (Russian translation, Gosudarstv. Izdat. Inostrannoı̆ Literatury, Moscow, 1969). MR 84h:33001

3. S. Pawelke, Ein Satz vom Jacksonschen Typ für algebraische Polynome, Acta Sci. Math. (Szeged) 33 (1972), no. 3-4, 323-336. MR 58 \#1848

4. M. K. Potapov, Nekotorye neravenstva dlya polinomov $i$ ikh proizvodnykh, Vestnik Moskov. Univ. Ser. I Mat. Mekh. (1960), no. 2, 10-20. MR 23 \#A3412

5. — O strukturnykh i konstruktivnykh kharakteristikakh nekotorykh klassov funktsiu, Trudy Mat. Inst. Steklov. 131 (1974), 211-231, 247-248. MR 50 \#14195

6. - O priblizhenii algebraicheskimi mnogochlenami v integral'nou metrike s vesom Yakobi, Vestnik Moskov. Univ. Ser. I Mat. Mekh. (1983), no. 4, 43-52. MR 84i:41008

7. M. K. Potapov and F. M. Berisha, Approximation of classes of functions defined by a generalized $k$-th modulus of smoothness, East J. Approx. 4 (1998), no. 2, 217-241. MR 1638345 (99g:41007)

8. M. K. Potapov and V. M. Fedorov, O teoremakh Dzheksona dlya obobshchennogo modulya gladkosti, Trudy Mat. Inst. Steklov. 172 (1985), 291-298, 355. MR 86m:41012

9. N. Ya. Vilenkin, Spetsiyal'nye funktsii i teoriya predstavlenii grupp, "Nauka", Moscow, 1991. MR 93d:33013

M. K. Potapov, Department of Mechanics and Mathematics, Moscow State UniverSity, Moscow 117234, Russia

F. M. Berisha, Faculty of Mathematics and Sciences, University of Prishtina, Nëna Terezë 5, 10000 Prishtinë, Kosov0

E-mail address: faton.berisha@uni-pr.edu 